Revue québécoise de linguistique

\title{
La structure conceptuelle de la référence : vers une représentation indépendante de la modalité d'expression
}

\section{Christine Poulin}

Volume 23, numéro 1, 1994

Sémantique

URI : https://id.erudit.org/iderudit/603078ar

DOI : https://doi.org/10.7202/603078ar

Aller au sommaire du numéro

Éditeur(s)

Université du Québec à Montréal

ISSN

0710-0167 (imprimé)

1705-4591 (numérique)

Découvrir la revue

Citer cet article

Poulin, C. (1994). La structure conceptuelle de la référence : vers une représentation indépendante de la modalité d'expression. Revue québécoise de linguistique, 23(1), 17-33. https://doi.org/10.7202/603078ar
Résumé de l'article

Certains aspects du sens affectent la forme linguistique d'un énoncé mais non sa structure syntaxique. Dans cet article, je montre que l'alternance des formes simples et à déplacement référentiel en LSQ en est un exemple, et que cette alternance indique un changement de point de vue de la part du locuteur par rapport à l'entité dont il parle. De plus, l'exploitation de la différence dans la façon de référer à une entité semble varier selon la modalité d'expression utilisée par la langue (visuo-spatiale vs audio-orale). Il s'ensuit qu'une représentation plus abstraite par rapport aux propriétés purement liées à la modalité serait plus compatible avec les stratégies différentes des langues signées et des langues orales. 


\title{
LA STRUCTURE CONCEPTUELLE DE LA RÉFÉRENCE: VERS UNE REPRÉSENTATION INDÉPENDANTE DE LA MODALITÉ D'EXPRESSION ${ }^{1}$
}

\author{
Christine Poulin \\ Stanford University
}

\section{Introduction}

DuISQue nous cherchons à concevoir un modèle de la grammaire qui rende compte de toutes les langues naturelles, il peut être intéressant de voir si des propriétés fondamentales communes à toutes les langues peuvent se réaliser différemment selon le type de langue étudiée (signée vs orale). Par exemple, certains aspects du sens affectent la forme linguistique d'un énoncé, mais non sa structure syntaxique. L'alternance des pronoms $i l$ et $c e$, cf. Coppieters $(1982,1990)$ ou en et $y$, cf. Ruwet (1990) en français et l'opposition pronominal vs anaphore dans certains contextes de l'anglais en sont des exemples. Ces alternances semblent indiquer un changement de point de vue de la part du locuteur par rapport à l'entité dont il parle, cf. Cantrall (1974); Zribi-Hertz (1989). Il peut s'agir soit d'un point de vue intérieur, soit d'un point de vue extérieur par rapport à cette entité. Considérons l'exemple suivant tiré de Coppieters (1982):

(1) Jean se rendait enfin compte qu'il avait tout essayé et tout raté.

$\left\{C_{I l}^{\prime}\right\}$ était un homme pauvre désormais. Pourquoi avait-il eu tant de malchance?

En (1), l'utilisation de $i l$ indique le point de vue intérieur de Jean qui réalise qu'il est un pauvre homme. Sous cette interprétation, la proposition "Pourquoi..." exprime la colère et le désespoir de Jean. Par contre, l'emploi de 
ce représente le point de vue extérieur du locuteur sur la situation de Jean et, dans ce cas, la proposition "Pourquoi..." devient une question rhétorique. Cet exemple montre qu'en français, le locuteur peut choisir de référer à une entité d'un point de vue intérieur ou extérieur selon le pronom qu'il utilise.

Les alternances observées en français et en anglais impliquent une différence dans la façon de faire référence à une entité. Dans cet article, je montrerai que, de la même façon, l'alternance des formes simples et à déplacement référentiel (ou jeu de rôle) permet d'exprimer un choix de points de vue de la part du signeur en langue des signes québécoise (LSQ). Nous verrons que l'exploitation de cette distinction au niveau de la référence semble varier selon la modalité utilisée par la langue (visuo-spatiale vs audio-orale) qui oppose simultanéité à linéarité. En effet, dans les langues signées, pour exprimer un changement dans la façon de référer à une entité, un signe linguistique (au sens large d'unité linguistique formée d'un signifiant et d'un signifié) est maintenu parallèlement aux éléments lexicaux pendant toute la durée d'un énoncé. Par contre, dans les langues orales qui sont linéaires (i.e. limitées par une plus stricte linéarité temporelle de l'expressibilité), les possibilités d'exprimer des signes linguistiques simultanément sont beaucoup plus restreintes. Il s'ensuit que les langues orales doivent normalement adopter une stratégie différente de celle des langues signées qui leur permette de distinguer efficacement les changements dans la façon de référer à une entité, par exemple le choix des items lexicaux.

\section{Déplacement référentiel: description et fonctions}

Lorsque que le déplacement référentiel prend place, le référent d'un locus spatial de troisième personne peut être réassigné à un locus spatial de première personne. Lillo-Martin \& Klima (1990) utilisent le terme cadre de référence pour désigner l'arrangement des locus associés à leurs référents respectifs. Dans le discours, ce cadre de référence peut se déplacer, prenant ainsi la forme d'un jeu de rôle. Le déplacement référentiel exprime un déplacement du cadre de référence. Il en résulte que ce qui semble être un signe pronominal de première personne s'interprète en fait comme un signe pronominal de troisième personne.

De façon générale, le déplacement référentiel s'exprime par des modifications de l'expression faciale et/ou par un changement dans le rythme et la façon de signer qui sont associés au protagoniste avec lequel le signeur s'identifie, cf. Loew (1984); Lillo-Martin \& Klima (1990); Meier (1990); Padden (1990). Une autre caractéristique est la présence d'un changement dans la position du corps ou dans l'orientation des épaules, de la tête et/ou des yeux du 
signeur. Le corps du signeur peut se déplacer, à partir d'une position non marquée, vers la localisation référentielle du protagoniste dont il assume le rôle. Ainsi, le corps peut se déplacer sur les côtés, cf. Loew (1984); Meier (1990); Padden (1990) ou légèrement en avant, cf. Padden, (1986, 1990). Par ailleurs, lorsque le signeur établit un locus pour situer un référent auquel il veut s'identifier, cette information ne fait habituellement pas partie du déplacement référentiel en LSQ, c'est-à-dire que le signeur situe le référent dans l'espace avant de changer le cadre de référence.

Le déplacement référentiel occupe différentes fonctions dans le discours, qui sont illustrées par les exemples (2) à (10) ci-dessous. Cependant, avant de présenter ces exemples, il est nécessaire d'expliquer la notation utilisée dans les transcriptions pour indiquer la flexion du verbe ou la référence du pointé ${ }^{2}$ qui sont particulièrement complexes dans un déplacement référentiel. Selon cette notation, les chiffres $1,2,3$ sont des indices pragmatiques dont la valeur référentielle dépend du rôle que tient une entité dans l'énoncé en fonction du point de vue courant adopté par le signeur. Ainsi, l'indice 1 réfère à l'entité dont le signeur adopte le point de vue (ce qui n'implique pas que cette entité soit le sujet de conscience de l'énoncé). L'indice 2 réfère à l'entité avec laquelle interagit l'entité dont le signeur a adopté le point de vue. Finalement, l'indice 3 réfère à toute autre entité qui n'est ni 1 ni 2. Les lettres $a, b, c$ et $z$ sont des indices spatio-référentiels qui marquent la position d'un locus donné dans l'espace référentiel: $z$ représente le corps du signeur et $a, b, c$ représentent des loci autres que le corps du signeur. Lorsque l'espace est utilisé référentiellement, il existe une relation d'égalité référentielle entre un référent et son locus, cf. Friedman (1975), entre autres. De ce fait, les indices spatioréférentiels indiquent la flexion du verbe pour la personne. Les lettres grecques $a, B$ et $\partial$ sont des indices spatio-topographiques qui marquent la localisation dans l'espace topographique. Dans une utilisation topographique de l'espace, un référent $i$ est considéré comme étant à une localisation $a$, cf. Liddell (1990). Ainsi, les indices spatio-topographiques rendent compte de la flexion du verbe pour la localisation (ce qui correspond aux rôles thématiques de source et de but). Finalement, les lettres $i, j, k$ sont des indices référentiels au sens propre qui sont associés à une entité donnée du discours et qui ne varient pas pendant le discours.

Le déplacement référentiel a été communément décrit comme étant comparable au discours direct dans les langues orales, c'est-à-dire lorsqu'un locuteur / signeur rapporte les paroles d'une tierce personne, cf. Ahlgren (1990); Engberg-Pederson (1992); Loew (1984); Lillo-Martin \& Klima (1990); Meier 
(1990); Padden (1986, 1990). Les séquences présentées en (2), (3) et (4) sont des exemples de rapport de paroles en LSQ. ${ }^{3}$

(2)

$\mathrm{DR}_{\mathrm{i}}$

PTE $_{3 \mathrm{ai}}$ FEMME $_{\mathrm{i}}$ ABANDONNER PTE $1 \mathrm{zi}$ S'EN-ALLER

'La femme: "J'abandonne, je m'en vais!»'

(3) ${ }_{1 \mathrm{zi}}$ DEMANDER $_{2 \mathrm{bj}}$ VERRE ${ }_{1 \mathrm{zi}} \mathrm{DONNER}^{\mathrm{V}} \mathrm{VERRE} \mathrm{b}_{2 \mathrm{j}}$

$\mathrm{DR}_{\mathrm{i}}$

'( Le serveur ${ }_{i}$ demande à Charlie Chaplin $)$ ): «Voulez-vous que je vous

donne un verre?»'

$\mathrm{DR}_{\mathrm{j}}$

neg PTE $_{1 z \mathrm{j}}$ PTE $_{3 \mathrm{ak}}$ neg OK GRANDE-BOUTEILLE $\mathrm{k}_{\mathrm{k}}$ BOIRE OK

'(Charlie Chaplin $)$ ): «Non, ça va, j'ai déjà une bouteille!»'

(4) PTE $_{1 \mathrm{zi}}$ VACANCES PTE pri $_{\text {PROMENADE PTE }}$ Fil FIS AVEC

'Mandela: "Je suis en vacances, en promenade avec mon fils.»'

$\mathrm{DR}_{\mathrm{j}}$

${ }_{1 \mathrm{z} j} \mathrm{ARRÊTER}_{2 \mathrm{ai}}$ ICI DEHORS

'Police: "Nous allons vous arrêter (si vous restez) ici, dehors!"'

L'utilisation du déplacement référentiel ne se limite pas au rapport de paroles. Elle s'étend au rapport d'action, cf. Ahlgren (1990); Engberg-Pederson (1992); Loew (1984); Meier (1990), des états, cf. Engberg-Pederson (1992); Meier (1990), des pensées ou des expériences, cf. Engberg-Pederson (1992). Les exemples (5), (6) et (7) illustrent le rapport d'action en LSQ:

(5) PTE ${ }_{3 \mathrm{ai}}$ REPASSER DÉPOSER-FER MARCHER $\mathrm{DR}_{\mathrm{i}}$

'Le tailleur, repassait, (il a vu Charlie Chaplin, ${ }_{j}$ ), il a déposé le fer et a marché vers lui.'

(6)

3aj PRENDRE FROMAGE $_{\mathrm{j}}$ METTRE-FROMAGE-DANS-TONNEAU ${ }_{32 \mathrm{j}}$

'(Elle $\left.{ }_{i}\right)$ a pris le fromage et l'a mis dans le tonneau.'

$\mathrm{DR}_{\mathrm{i}}$

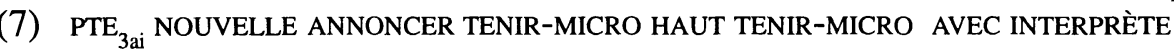
'La nouvelle présidente ${ }_{i}$ de Gallaudet, accompagnée d'une interprète, a pris le micro pour faire une annonce.'

Le rapport de pensées est illustré par l'exemple (8), où le signeur rapporte une réflexion que le protagoniste auquel il s'identifie se fait à lui-même. 
(8)
$\mathrm{DR}_{\mathrm{j}}$
PANTALON TROU ${ }_{\mathrm{k}}$ PTE $_{3 \mathrm{bk}}$ (geste) IMBÉCILE PTE $3 \mathrm{ai}$
'Il y a un trou dans le pantalon... quel imbécile!'

Finalement, le rapport d'états physiques ou émotionnels est représenté par les phrases (9) et (10) (qui comprend également un rapport de pensées):

(9) PROPRIÉTAIRE ${ }_{\mathrm{i}} \overline{\text { 1zi }}_{\text {REGARDER }_{2 \mathrm{j}} \text { ÊTRE-CONTRARIÉ }}$

'Le propriétaire ${ }_{i}$ regardait Charlie Chaplin, il ${ }_{i}$ était contrarié.'

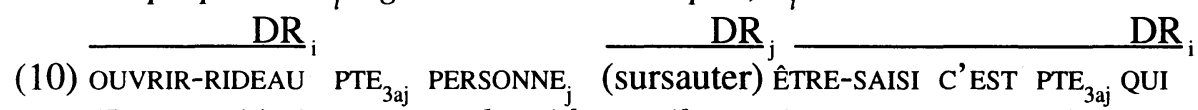

'Le propriétaire a ouvert les rideaux, il y avait une personne qui a

sursauté. Le propriétaire a été saisi: "C'est qui?», s'est-il demandé.'

Les différents types de discours décrits ci-dessus en LSQ (rapports de paroles, d'actions, de pensées et d'états), impliquent tous un transfert d'indices référentiels. Toutefois, nous remarquons que la forme linguistique des structures semble demeurer la même indépendamment du type d'énoncé. Comment alors distinguer le rapport de paroles du rapport d'action et ainsi de suite? L'analyse sommaire des données semble indiquer d'une part, que l'oralisation ${ }^{4}$ serait peut-être impliquée pour distinguer le discours direct d'une autre forme de discours. En effet, il semble que l'oralisation est utilisée lorsque le signeur s'adresse à un interlocuteur, qu'il soit réel (forme neutre) ou fictif (déplacement référentiel) comme en témoigne l'exemple (11) où le verbe REPASSER n'est pas oralisé lorsqu'il s'agit d'un rapport d'action (première partie du DR) alors qu'il l'est lorsque les paroles sont rapportées (deuxième partie du DR). Dans la transcription, les signes oralisés sont indiqués par les lettres or qui leur sont superposées.

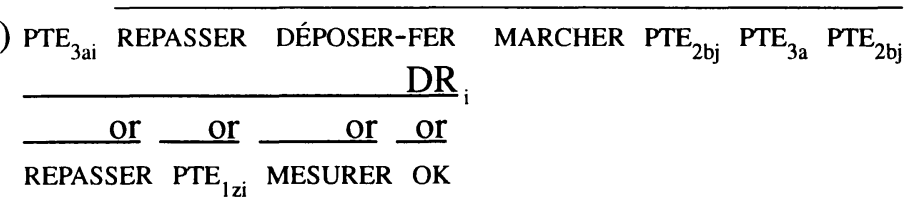

'Le tailleur repassait, (il a vu Charlie Chaplin, ${ }_{j}$ ), il a déposé le fer et a marché vers lui $i_{j}$ " Toi tu vas repasser et moi je vais mesurer OK?»'

L'oralisation pourrait aussi être utilisée lors du rapport de pensées, puisque le signeur s'adresse alors à lui-même. D'autre part, l'utilisation du PTE dans un déplacement référentiel semble forcer l'interprétation de discours direct, comme l'a souligné Meier (1990) pour la langue des signes américaine 
(ASL) (cependant, l'absence de $\mathrm{PTE}_{1}$ n'implique pas nécessairement qu'il ne puisse s'agir de discours direct). La LSQ disposerait donc de deux moyens pour distinguer un rapport de paroles (et de pensées) d'un autre type de rapport. Toutefois, il ne s'agit ici que d'une hypothèse qui nécessite des travaux plus approfondis.

\section{Déplacement référentiel et point de vue narratif}

Dans une narration, nous observons que certains énoncés peuvent prendre deux formes. Ainsi, pour décrire un événement où un ou plusieurs protagonistes interagissent, le signeur peut rapporter l'action et référer aux participants en utilisant soit le déplacement référentiel, soit la forme simple. La possibilité d'utiliser une forme ou l'autre pour référer à la même entité suggère qu'une différence existe dans la façon de faire référence à cette entité.

Dans cette section, je montrerai qu'une des fonctions du déplacement référentiel en LSQ est d'exprimer un changement de point de vue de la part du signeur, de la même façon que l'alternance des pronoms $i l$ et $c e$ en français, ou du pronominal et de l'anaphore dans certaines constructions de l'anglais.

\subsection{Alternance forme simple / déplacement référentiel}

Considérons les paires de phrases suivantes:

(12) a. PTE 3ai $_{\text {REPASSER DÉPOSER-FER MARCHER }}$

'Le tailleur ${ }_{i}$ repassait, (il a vu Charlie Chaplin ${ }_{j}$ ), il a déposé le fer et a marché vers lui,

b. PTE ${ }_{3 a i}$ REPASSER DÉPOSER-FER MARCHER

'Le tailleur ${ }_{i}$ repassait, (il a vu Charlie Chaplin, ${ }_{j}$, il a déposé le fer et a marché vers lui,

(13) a. PTE 3ai $_{\text {NOUVELLE ANNONCER TENIR-MICRO HAUT TENIR-MICRO AVEC }}$ INTERPRÈTE

'La nouvelle présidente ${ }_{i}$ de Gallaudet, accompagnée d'une interprète, a pris le micro pour faire une annonce.'

b. PTE ${ }_{3 \mathrm{a}}$ NOUVELLE ANNONCER TENIR-MICRO HAUT TENIR-MICRO AVEC INTERPRÈTE

'La nouvelle présidente de Gallaudet, accompagnée d'une interprète, a pris le micro pour faire une annonce.' 


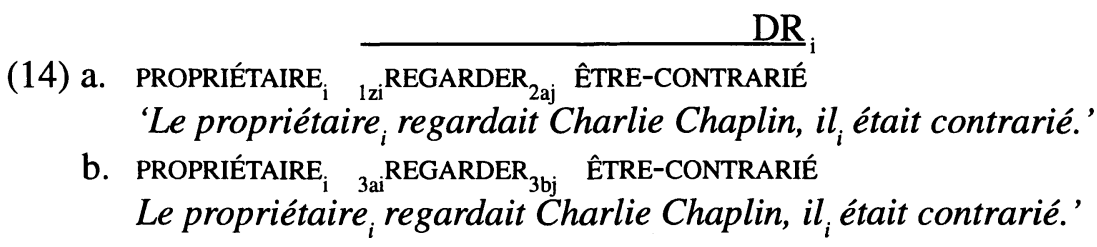

Lorsqu'ils ont été questionnés à propos de leurs intuitions sur l'existence d'une différence sémantique entre des paires de phrases du type de (12) à (14), les consultants ont indiqué que l'utilisation du déplacement référentiel est favorisée dans le discours lorsque le signeur veut donner plus d'informations et de détails sur un événement donné. De plus, ils indiquent que le déplacement référentiel exprime l'état émotionnel ou les sentiments intérieurs du participant du discours avec lequel le signeur établit l'empathie. Ainsi, la forme avec déplacement référentiel ( $+\mathrm{DR})$ exprimerait un point de vue intérieur de la part du signeur par rapport à l'entité (le signeur s'identifie à un participant), alors que l'utilisation de la forme simple (-DR) exprimerait plutôt un point de vue extérieur par rapport à l'entité (point de vue neutre ou celui du signeur).

\subsection{Portée du modifieur}

Le comportement non manuel a, entre autres fonctions, celle de modifieur de phrase ou de constituant, cf. Baker \& Padden (1978). Si nous considérons les exemples (15), (16) et (17) ci-dessous, nous observons que le comportement non manuel (plus précisément l'expression du visage du signeur) a une portée différente selon qu'il est utilisé dans une forme +DR ou -DR.

(15) a. VOLEUR PTE $_{3 \mathrm{ai}}$ HOMME $_{\mathrm{j}}$ PTE $_{3 \mathrm{bj}}$ 1zi FRAPPER-AVEC-BÂTON $_{2 \mathrm{bj}}$ 'En colère, le voleur a frappé l'homme avec un bâton.'

b. VOLEUR ${ }_{\mathrm{i}}$ PTE $_{3 \mathrm{ai}}$ HOMME $_{\mathrm{j}}$ PTE $_{3 \mathrm{bj}} \frac{\text { 'colère' }}{\text { 3ai }_{\text {FRAPPER-AVEC-BÂTON }}}$ 'Ça m'a mis en colère quand le voleur a frappé l'homme avec un bâton.'

$\mathrm{DR}_{\mathrm{i}}$ 'colère' 
b. ÉTUDIANT PTE $_{3 \mathrm{ai}}$ PROFESSEUR $_{\mathrm{j}}$ PTE $_{3 \mathrm{bj}} \frac{\text { 'nerveux' }}{{ }_{3 \mathrm{ai}} \text { POSER-UNE-QUESTION }_{3 \mathrm{bj}}}$ 'Ça m'a rendu nerveux quand l'étudiant a posé une question au professeur.'

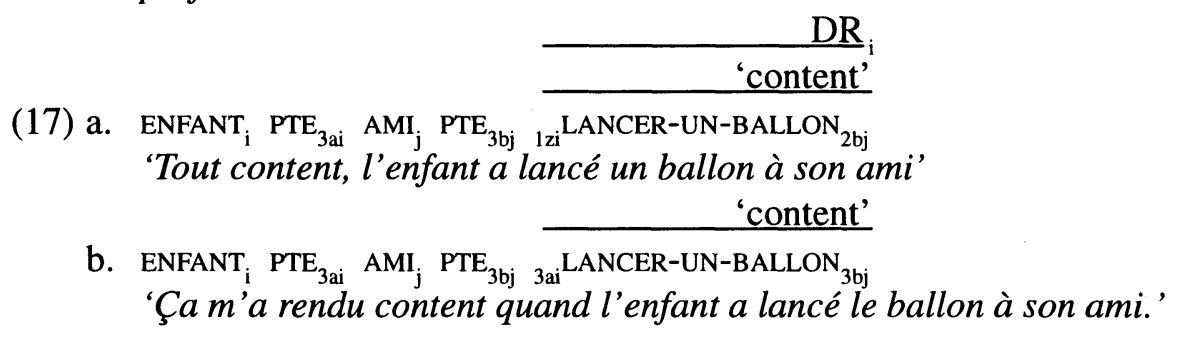

L'expression du visage dans une forme -DR exprime un jugement, une critique ou une émotion de la part du signeur par rapport à l'événement qu'il décrit, alors qu'une expression du visage semblable réalisée dans une forme +DR exprime plutôt les sentiments intérieurs du protagoniste du discours avec lequel le signeur s'identifie. Ces observations sur la portée du modifieur en fonction du déplacement référentiel vont dans le sens d'un point de vue différent exprimé par le signeur, selon qu'il utilise ou non un déplacement référentiel pour référer à une entité donnée.

\subsection{Représentation du déplacement référentiel}

Considérons les deux équivalents en LSQ de la phrase 'Le voleur a frappé l'homme avec un bâton' donné en (18a) et (18b), le changement de forme suggère qu'une différence existe dans la façon avec laquelle le signeur réfère aux participants du discours.

(18) a. VOLEUR PTE ${ }_{3 \mathrm{ai}}$ HOMME PTE Pbj $_{\text {1zi }} \frac{\mathrm{DR}_{\mathrm{i}}}{\text { FRAPPER-AVEC-BÂTON }_{2 \mathrm{bj}}}$

'Le voleur a frappé l'homme avec un bâton'

b. VOLEUR PTE ${ }_{3 \mathrm{ai}}$ HOMME PTE ${ }_{3 \mathrm{bj}}$ 3ai $^{\text {FRAPPER-AVEC-BÂTON }}{ }_{3 \mathrm{bj}}$ 'Le voleur a frappé l'homme avec un bâton'

De ce fait, nous remarquons que l'ajout d'un commentaire neutre ou impliqué par rapport au contenu de l'événement décrit a un effet différent selon que ce commentaire suit une forme $+D R$ ou $-D R$. Ainsi, si nous considérons les phrases (18a) et (18b), nous observons que l'ajout du commentaire 'PTE ${ }_{3 a i}$ POLICE ARRIVER AVOIR-PEUR' (il avait peur que la police arrive), dont le contenu est émotionnellement relié au contenu de l'événement décrit n'a pas d'effet inhabituel lorsque ce commentaire suit une forme -DR comme le montre 
l'exemple en (19a). Par contre, lorsqu'il est ajouté à une forme +DR, une anomalie est créée si le commentaire est réalisé à l'intérieur du déplacement référentiel comme en (19b).

(19) a. VOLEUR PTE $_{3 \mathrm{ai}}$ HOMME $_{\mathrm{j}}$ PTE $_{3 \mathrm{bj}}$ 3ai $_{\mathrm{a}}$ FRAPPER-AVEC-BÂTON $_{3 \mathrm{bj}}$ PTE $_{3 a i}$ POLICE ARRIVER AVOIR-PEUR

'Le voleur ${ }_{i}$ a frappé l'homme avec un bâton. $\mathrm{Il}_{i}$ avait peur que la police arrive.'

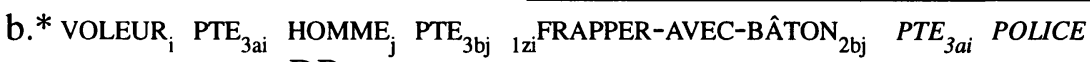
$\mathrm{DR}_{\mathrm{i}}$

ARRIVER AVOIR-PEUR

'Le voleur ${ }_{i}$ a frappé l'homme avec un bâton. $I l_{i}$ avait peur que la police arrive.'

Il serait possible d'argumenter que l'anomalie de (19b) est causée uniquement par des facteurs structuraux, c'est-à-dire qu'un conflit existe entre les indices spatiaux qui réfèrent au voleur (localisation $3 a$ vs la référence apparente à la première personne dans le déplacement référentiel, $1 z$ ). Toutefois, d'autres données dans notre corpus indiquent qu'un tel doublement d'indices spatiaux apparemment incompatibles est acceptable à l'intérieur d'un déplacement référentiel, comme le montre la phrase (20).

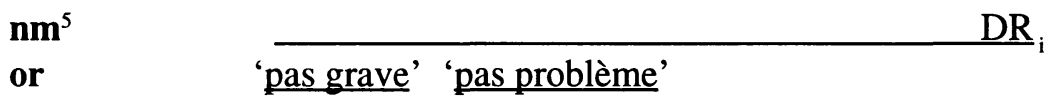

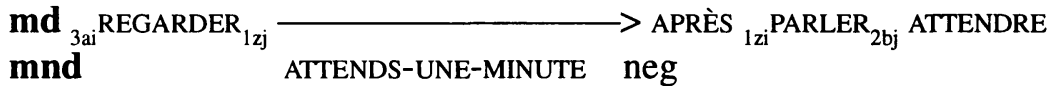

'(Le professeur) m'a regardé et a dit: "Attends une minute, ce n'est pas grave, ce n'est pas un problème... Je vais t'en parler plus tard».'

En (20), il s'agit d'un encodage parallèle où plusieurs éléments lexicaux sont réalisés simultanément. Le signeur signe ' ${ }_{3 \mathrm{ai}} \mathrm{REGARDER}_{1 \mathrm{zj}}{ }^{\text {'(il m'a regardé) }}$ avec la main dominante, il tient ce signe et, au même moment, il entre dans un déplacement référentiel où il s'identifie avec le professeur. La phrase (20) est représentée en (21), où nous observons un domaine référentiel matrice et un domaine référentiel dépendant (par rapport à cette matrice). La matrice représente le domaine référentiel de base où les éléments sont situés dans l'espace et dans le temps. Ce domaine peut contenir un événement, mais ce n'est pas obligatoire. En (21), la matrice contient l'événement ' ${ }_{3 \mathrm{ai}}$ REGARDER $_{1 \mathrm{zj}}$ '. Le domaine référentiel dépendant correspond au déplacement référentiel effectué à partir de la matrice de base. Dans ce cas, le domaine dépendant contient l'évé- 
nement 'ATTENDRE-UNE-MINUTE APRÈS ${ }_{1 \mathrm{zi}}$ PARLER $_{2 \mathrm{bj}}$ ATTENDS' (représenté par ${ }_{1 \mathrm{i}}$ PARLER $_{2 \mathrm{bj}}$ en (21)).

(21)

Domaine référentiel matrice

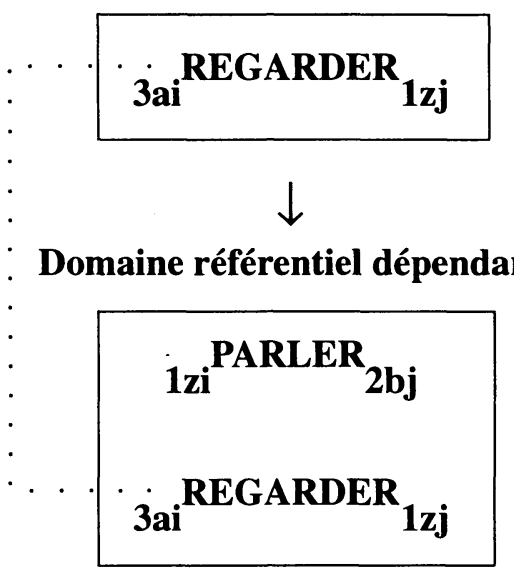

Dans cet énoncé, la référence à la troisième personne dans la matrice (3ai) correspond à celle de la première personne dans le déplacement référentiel (1zi). Du point de vue de la première personne dans le déplacement référentiel, la personne regardée est la deuxième personne. Dans le domaine du déplacement référentiel, nous observons que l'indice de localisation $z$ est lié avec deux indices référentiels distincts, $i$ et $j$. De plus, nous observons que l'indice référentiel $i$ est lié à deux indices spatiaux différents $a$ et $z$ (il en est de même pour l'indice $j$ qui est lié à la fois aux indices $b$ et $z$ ). Ce qui semble être un conflit entre les indices référentiels et spatiaux est acceptable dans la phrase (20) parce que, dans le domaine du déplacement référentiel, les différents indices ont accès à leur antécédent. En effet, les indices du verbe REGARDER tel que tenu dans le déplacement référentiel peuvent accéder à leur antécédent dans la matrice parce que ce verbe retient son contenu événementiel tant qu'il est tenu et quoique sa position d'origine soit dans la matrice. Toutefois, en (21), le fait que le signe REGARDER soit tenu plutôt que simultanément réalisé avec le déplacement référentiel peut avoir un effet sur la grammaticalité du doublement des indices référentiels. Des recherches plus approfondies sur cette question sont nécessaires. 
Considérons maintenant la phrase (19b) qui est représentée en (22) par le domaine matrice 'VOLEUR ${ }_{\mathrm{i}}$ PTE $_{3 \mathrm{ai}}$ HOMME $_{\mathrm{j}}$ PTE $_{3 \mathrm{bj}}$ ' et le domaine dépendant ' ${ }_{1 \mathrm{ii}}$ FRAPPER-AVEC-BÂTON ${ }_{2 \mathrm{bj}}$ PTE $_{3 \mathrm{ai}}$ POLICE ARRIVER AVOIR-PEUR' (représenté par ${ }_{1 \mathrm{zi}}$ FRAPPER-AVEC-BÂTON ${ }_{2 \mathrm{bj}}$ PTE $_{3 \mathrm{ai}}$ en (22)).

Domaine référentiel matrice

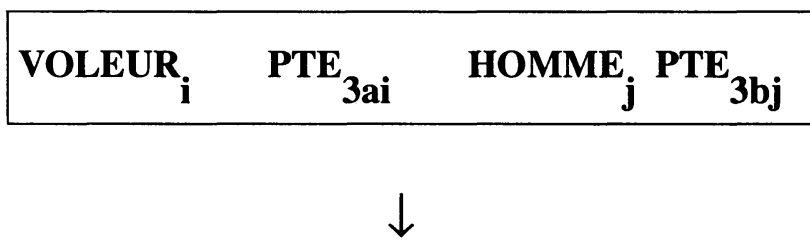

Domaine référentiel dépendant

$$
\text { 1zi }^{\text {FRAPPER-AVEC-BÂTON }_{2 b j}} \text { PTE }_{3 a i}
$$

Dans cet énoncé, nous observons qu'à l'intérieur du domaine du déplacement référentiel, la personne à qui réfère l'indice référentiel $i$ est liée avec deux indices spatiaux différents: $z$ et $a$. Nous nous attendrions à ce que ce doublement d'indices soit grammatical comme nous l'avons vu en (20). Toutefois, la phrase (19b) est inacceptable. Ceci pourrait être expliqué par le fait que l'indice pragmatique de troisième personne et l'indice spatial $a$ ne peuvent pas être interprétés comme étant liés avec l'indice référentiel $i$ parce qu'ils n'ont pas d'antécédent à l'intérieur du domaine du déplacement référentiel. La phrase (19b) est donc éliminée et la phrase (23) ci-dessous, où le pronom de troisième personne $\left(\mathrm{PTE}_{3}\right)$ n'est pas réalisé, est tout à fait acceptable. Par ailleurs, le fait que le PTE $_{3}$ ne soit pas réalisé implique peut-être qu'il existe une relation de coordination entre les deux phrases, ce qui est plus serré comme relation. À cet égard, les langues signées se rapprocheraient des langues de type pro-drop.

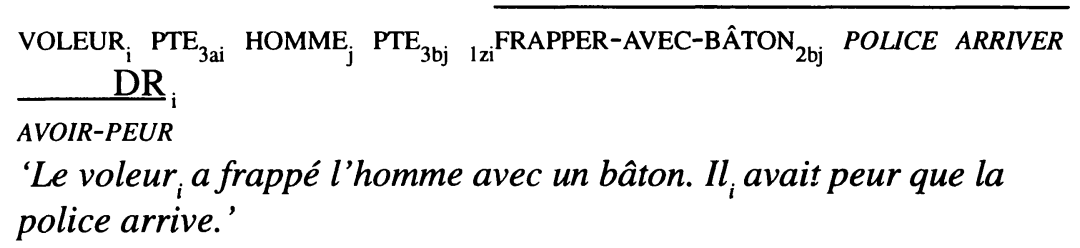

Considérons maintenant les phrases (24a) et (24b). 
(24) a.* VOLEUR ${ }_{\mathrm{i}}$ PTE $_{3 \mathrm{ai}}$ HOMME $_{\mathrm{j}}$ PTE $_{3 \mathrm{bj}} \overline{\text { 1zi } \text { FRAPPER-AVEC-BÂTON }_{2 \mathrm{bj}} \text { PTE }_{3 \mathrm{ai}} \text { HABITER }}$ $\mathrm{DR}_{\mathrm{i}}$ SHERBROOKE

'Le voleur ${ }_{i}$ a frappé l'homme avec un bâton. $I_{i}$ habite à Sherbrooke.'

b.\# VOLEUR ${ }_{\mathrm{i}}$ PTE $_{3 \mathrm{ai}}$ HOMME $_{\mathrm{j}}$ PTE $_{3 \mathrm{bj}} \overline{\text { 1zi }_{\text {FRAPPER-AVEC-BÂTON }} \text { Hbj }^{\text {HABITER }}}$ $\frac{\text { DR }}{\text { SHERBROOKE }}_{\mathrm{i}}$

'Le voleur ${ }_{i}$ a frappé l'homme avec un bâton. Il ${ }_{i}$ habite à Sherbrooke.'

Nous observons que l'ajout du commentaire 'Il (le voleur) habite à Sherbrooke', qui est neutre par rapport au contenu de l'événement, crée une anomalie s'il est signé à l'intérieur du déplacement référentiel. En (24a) l'agrammaticalité de la phrase s'explique par le conflit qui existe entre les indices spatiaux (localisation $3 a$ vs $1 z$ ) comme les exemples (19) à (23) cidessus l'ont démontré. Cependant, en (24b), l'anomalie de la phrase ne peut être attribuée qu'à l'incongruité de la neutralité du commentaire par rapport à l'implication personnelle du signeur et non à des facteurs structuraux. De ce fait, la phrase n'est pas agrammaticale, mais plutôt inacceptable (ce qui est représenté par un \#), puisque qu'elle est structuralement bien formée.

Toutefois, l'ajout de ce même commentaire à l'extérieur du déplacement référentiel est tout à fait acceptable, comme le mon tre l'exemple en (25a). Il en va de même lorsqu'un commentaire impliqué par rapport à l'événement est ajouté à l'extérieur du déplacement référentiel (exemple 25b).

(25) a. VOLEUR PTE $_{3 \mathrm{ai}}$ HOMME $_{\mathrm{j}}$ PTE $_{3 \mathrm{bj}} \frac{\mathrm{DR}_{\mathrm{i}}}{\text { 1zi }_{\mathrm{iz}} \text { FRPPER-AVEC-BÂTON }_{2 \mathrm{jj}}}$ PTE $_{3 a i}$ HABITER SHERBROOKE

'Le voleur ${ }_{i}$ a frappé l'homme avec un bâton. Il ${ }_{i}$ habite à Sherbrooke.' $\mathrm{DR}_{\mathrm{i}}$

b.? VOLEUR ${ }_{\mathrm{i}}$ PTE $_{3 \mathrm{ai}}$ HOMME $_{\mathrm{j}}$ PTE $_{3 \mathrm{bj}}{ }_{\text {1zi }}^{\text {FRAPPER-AVEC-BÂTON }}{ }_{2 \mathrm{bj}}$ PTE $3 a i$ POLICE ARRIVER AVOIR-PEUR

'Le voleur ${ }_{i}$ a frappé l'homme avec un bâton. $I_{i}$ avait peur que la police arrive.'

Cependant, par opposition à l'exemple (23), où le commentaire est à l'intérieur du déplacement référentiel, la phrase en (25b) semble être moins naturelle, c'est-à-dire que les signeurs montrent une préférence pour une phrase du type de (23). Ceci s'explique par le fait que si le signeur raconte un événement 
en se plaçant du point de vue du voleur, il est bizarre qu'il quitte ce point de vue pour décrire les émotions du voleur.

En résumé, nous avons vu, avec les exemples présentés de (18) à (25), que l'ajout d'un commentaire à une forme $+\mathrm{DR}$ a des effets différents selon que ce commentaire est neutre ou impliqué par rapport au contenu de l'événement décrit, et que l'acceptabilité de l'énoncé varie selon que le commentaire est signé à l'intérieur ou à l'extérieur du déplacement référentiel. Nous avons observé qu'un commentaire neutre est inacceptable à l'intérieur d'un déplacement référentiel alors qu'un commentaire impliqué est acceptable à condition toutefois de respecter le domaine de référence des indices. Ceci appuie l'hypothèse que le choix de la forme linguistique d'un énoncé exprime un choix de point de vue de la part du signeur et qu'une alternance impliquant un déplacement référentiel remplit cette fonction en LSQ.

L'analyse présentée ci-dessus pour l'interprétation des indices et de leurs antécédents à l'intérieur du domaine du déplacement référentiel nous amène à une représentation en termes de domaine référentiel telle qu'illustrée en (26).

\section{Domaine référentiel matrice}

(point de vue du narrateur)

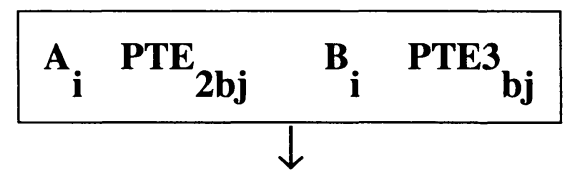

Domaines référentiels dépendants

(point de vue des actants)

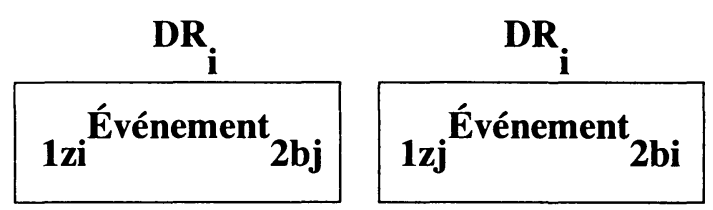

Dans un énoncé, le domaine de référence matrice consiste en l'établissement du domaine référentiel de base. Il s'agit d'un cadre de référence permanent où les éléments sont situés référentiellement dans l'espace et dans le temps (en (26) les actants A et B sont situés dans la matrice). La matrice exprime le point de vue du narrateur et elle peut, par le fait même, avoir un contenu événementiel, mais ce n'est pas obligatoire. À partir de cette matrice, le signeur peut décider de déplacer le cadre de référence s'il veut exprimer le point de vue de l'un des actants du discours. L'interprétation de ce nouveau cadre (ou domaine) 
de référence dépend donc de l'établissement des référents dans la matrice. Le signeur peut donc référer aux différents actants du discours en adoptant alternativement leurs cadres de référence respectifs.

\section{Conclusion}

En LSQ, dans les narrations, certaines constructions (essentiellement les actions et les états) peuvent prendre deux formes, c'est-à-dire que le signeur réfère aux actants du discours en utilisant soit le déplacement référentiel, soit la forme simple. Ceci suggère qu'il est possible pour le signeur de référer différemment à une même entité et qu'il peut choisir d'y faire référence en adoptant un point de vue intérieur ou extérieur relativement à cette entité. Cette hypothèse est renforcée par le fait que l'ajout, à l'intérieur d'un déplacement référentiel, d'un commentaire neutre par rapport au contenu de l'événement décrit est inacceptable, alors que l'ajout d'un commentaire impliqué est tout à fait naturel. Ainsi, dans certains cas, le déplacement référentiel aurait pour fonction d'exprimer un choix de points de vue de la part du signeur en ce qui concerne l'entité à laquelle il réfère et les conditions régissant ce choix ne s'établissent pas en termes de facteurs structuraux, mais plutôt en termes de points de vue à exprimer. Sous ce rapport, la distinction qui existe entre les formes simples et à déplacement référentiel en LSQ se rapproche beaucoup de ce qui a été discuté à propos de la distribution des pronoms il et $c e, \mathrm{cf}$. Coppieters $(1982,1990)$ en français.

Si nous observons les alternances impliquant un choix d'items lexicaux en français, nous remarquons que certains aspects du sens affectent la forme linguistique d'un énoncé, mais non sa structure syntaxique. Ces observations suggèrent que les notions sémantiques doivent être séparées en au moins deux types, cf. Bouchard (1992). Selon Bouchard, ces phénomènes relèvent de l'expression de la L-sémantique qu'il distingue de la G-sémantique. Ainsi, la L-sémantique comprend les aspects du sens qui affectent les choix linguistiques sans que ces aspects soient représentés dans la grammaire formelle. Par contre, la G-sémantique définit les propriétés grammaticales formelles (par exemple, les réalisations syntaxiques possibles d'un argument). À ces deux types de sémantique, Bouchard oppose la S-sémantique qui inclut tous les aspects du sens qui sont situationnels et qui, par le fait même, sont indépendants de la Grammaire. Par exemple, le fait que le déplacement référentiel puisse exprimer indifféremment un rapport de paroles, d'actions, de pensées ou d'états relève de la S-sémantique et n'a aucun effet sur la structure de la Grammaire. 
Les alternances impliquant le déplacement référentiel en LSQ semblent se comporter de la même façon, entre autres, que l'opposition des pronoms $i l$ et $c e$, en ce sens que le choix de la forme linguistique de l'énoncé ne peut être imputé à des facteurs structuraux, mais bien à des facteurs sémantiques et plus précisément à la manière de faire référence à une entité. Toutefois, une représentation du déplacement référentiel s'impose pour mieux comprendre les processus impliqués.

La situation référentielle et événementielle des éléments du discours de même que l'exploitation de la différence dans la façon de référer à une entité semblent varier selon la modalité d'expression utilisée par la langue (visuospatiale vs audio-orale) qui oppose simultanéité à linéarité. Dans les langues signées, pour exprimer une variation dans la référence à une entité, un signe linguistique peut être maintenu parallèlement aux items lexicaux pendant toute la durée d'un énoncé (par exemple, le déplacement référentiel). Par contre, dans les langues orales qui sont linéaires, les possibilités d'exprimer des signes linguistiques simultanément sont beaucoup plus restreintes. Il s'ensuit que les langues orales doivent normalement adopter une stratégie différente de celle des langues signées qui leur permette de distinguer efficacement les changements dans la façon de référer à une entité, comme le choix des items lexicaux (par exemple, l'opposition des pronoms $i l$ et $c e$ en français).

Les données présentées dans cet article montrent qu'une représentation basée exclusivement sur l'étude des langues orales risque d'être trop restrictive parce que certaines propriétés dérivées de la modalité physique dans lesquelles ces langues sont encodées (par exemple, une forte linéarisation) pourraient être faussement attribuées à la Grammaire, ce qui serait incompatible avec le fait que les langues signées permettent à des éléments d'être exprimés simultanément. Il serait intéressant de mieux décrire ces phénomènes, notamment en tentant de comprendre précisément les propriétés conceptuelles impliquées de façon à déterminer ce qui doit être exclu des représentations formelles, pour ensuite proposer une représentation qui soit plus abstraite par rapport aux propriétés purement liées à la modalité et par conséquent compatible avec les stratégies différentes des langues signées et des langues orales.

\section{Notes}

1. Cet article reprend en partie une communication intitulée «On narrative discourse and point of view in Quebec Sign Language», présentée dans le cadre du Fourth International Conference on Theoretical Issues in Sign Language Research à l'Université de Californie, San Diego en août 
1992, et qui paraîtra dans Language, gesture and space, publié sous la direction de Karen Emmorey et Judith Reilly. Je remercie Denis Bouchard, Colette Dubuisson, Marie Labelle, Christopher Miller, Dominique Pinsonneault et Anne Rochette pour leurs commentaires et suggestions, ainsi que mes consultants pour la LSQ Linda Lelièvre, Michel Lelièvre et Jules Desrosiers, pour leur collaboration.

2. Le pointé est un signe pronominal de 1ère, 2e ou 3e personne. Lorsqu'il est dirigé vers un locus donné, il permet d'établir une relation référentielle entre une entité et un locus.

3. Le sens en français de chaque signe est rendu approximativement par des gloses en majuscules. Lorsque la glose d'un signe exige plus d'un mot français, ces mots sont reliés entre eux par des traits d'union. Par convention, on représente à la forme infinitive les gloses des verbes et au singulier celles des substantifs. La glose PTE correspond à pointé. Les lettres DR correspondent à déplacement référentiel et l'indice indique le référent sur lequel porte le DR. Le trait horizontal continu superposé aux gloses spécifie la durée du déplacement référentiel par rapport au temps de réalisation des signes.

4. L'oralisation réfère au mouvement des lèvres qui reproduisent complètement ou partiellement des mots de la langue orale avec laquelle la langue signée est en contact, en l'occurrence, le français.

5. Les abréviations $n m$, or, $m d$, mnd signifient respectivement non manuel, main dominante, et main non dominante. Généralement la main dominante est celle qui exécute les signes ne demandant qu'une seule main et/ou qui exerce le mouvement dans les signes où une seule main bouge. Il s'agit le plus souvent de la main droite pour les droitiers et de la gauche pour les gauchers. L'autre main est la main non dominante. Un trait horizontal discontinu suivant une glose indique que ce signe est tenu.

\section{Références}

Ahlgren, I. (1990) «Deictic pronouns in Swedish and Swedish Sign Language», in Fischer \& Siple (réd.), Theoretical Issues in Sign Language Research I: Linguistics, Chicago, University of Chicago Press, p. 167-174.

BAKER, C. \& C. PADDEN (1978) «Focusing on the nonmanual components of American Sign Language», in Understanding language through sign language research, New York, Academic Press, p. 27-57.

BouchARD, D. (1992) The semantics of syntax, manuscrit, Université du Québec à Montréal.

CANTRALL, W. (1974) Viewpoint, reflexives and the nature of noun phrases, La Haye, Mouton.

COPPIETERS, R. (1982) «Descriptions and attitudes: The problem of reference to individuals», Studies in Language, 6, 1, p. 1-22.

COPPIETERS, R. (1990) Individual, thematic roles and semantic interpretation, manuscrit, Pomona College. 
ENGBERG-PEDERSON, E. (1992) «Speech reports, reported thoughts, and other kinds of reports», communication présentée au Fourth International Conference on Theoretical Issues in Sign Language Research, University of California, San Diego, 5-8 août.

FriedMAN, L. (1975) «Space, time, and person reference in American Sign Language», Language, vol. 51, p. 940-961.

LiDDELL, S.K. (1990) «Four functions of a locus: Reexamining the structure of space in ASL», in Lucas (réd.), Sign Language Research: Theoretical Issues, Washington, Gallaudet University Press, p. 176-198.

Lillo-Martin, D. \& E.S. Klima (1990) «Pointing out differences: ASL pronouns in syntactic theory», in Fischer \& Siple (réd.), Theoretical Issues in Sign Language Research I: Linguistics, Chicago, University of Chicago Press, p. 191-210.

Loew, R. (1984) Roles and reference in American Sign Language: A developmental perspective, thèse de doctorat, University of Minnesota.

MeieIR, R. (1990) «Person deixis in American Sign Language», in Fischer \& Siple (réd.), Theoretical Issues in Sign Language Research I: Linguistics, Chicago, University of Chicago Press, p. 175-190.

PADDEN, C. (1986) «Verbs and role-shifting in ASL», in Padden (réd.), Proceedings of the Fourth National Symposium on Sign Language Research and Teaching, Silver Spring, Maryland, National Association of the Deaf, p. 44-57.

PADDEN, C. (1990) «The relation between space and grammar in ASL verb morphology», in Lucas (réd.), Sign Language Research: Theoretical Issues, Washington, Gallaudet University Press, p. 118-132.

RUwET, N. (1990) «En et $y$ : deux clitiques pronominaux antilogophoriques», Langages, vol. 97, p. 51-81.

ZRIBI-HERTZ, A. (1989) «Anaphor binding and narrative point of view: English reflexive pronouns in sentence and discourse», Language, vol. 65, $\mathrm{n}^{\circ}$ 4, p. 695-727. 